Proceedings of the 1999 IEEE

International Workshop on Robot and Human Interaction

Pisa, Italy - September 1999

\title{
Experiments with a Steady Hand Robot in Constrained Compliant Motion and Path Following
}

\author{
Rajesh Kumar ${ }^{1}$, Patrick Jensen ${ }^{2}$, Russell H Taylor ${ }^{1}$ \\ Johns Hopkins University \\ Baltimore, Maryland, USA \\ ${ }^{1}$ Department of Computer Science, Whiting School of Engineering \\ ${ }^{2}$ Wilmer Eye Institute, Johns Hopkins Medical Institutions
}

\begin{abstract}
We consider the problem of cooperative manipulation to improve positioning and path following abilities of humans. Using a specially designed actuated manipulator and "steady hand" manipulation we report on compliant path following strategies and their experimental evaluation. Detecting lines and simple curve by processing images from an endoscope mounted on the robot, we traverse these curves autonomously, under direct user control, and in an augmented mode of user control. Anisotropic gains based on gradient information from the imaging reduce errors in path traversal.
\end{abstract}

\section{Introduction}

Several innovative surgical procedures such as treatment of occluded veins in the eye can be made possible through the use of "steady hand" manipulators by overcoming human sensorimotor limitations. An augmented procedure combines the precise positioning abilities of the robot and superior knowledge and experience of the surgeon that is difficult to encode in a machine for accurate administration of various treatments.

In addition to tool stabilization, the robot can remove tremor, performed scaled motions, enforce safety and retrace previous paths. To enforce safety, constraints can also be imposed to restrict the motion of the robot within a user-specified envelope around the detected path. The actions on meeting a constraint (stop, do not cross, ignore, inform user) can be programmed by the user. If the target location can be imaged and recognized using image processing, the robot can then "guide" the user towards the target by favoring motion in the desired direction.

This work reports experiments using a new "steadyhand" robot ([1]) developed at The Johns Hopkins University. This robotic system is designed to extend a human's ability to perform small-scale (sub-millimeter) manipulation tasks requiring human judgment, sensory integration and hand-eye coordination. The robot is operated using "steady hand" manipulation to provide

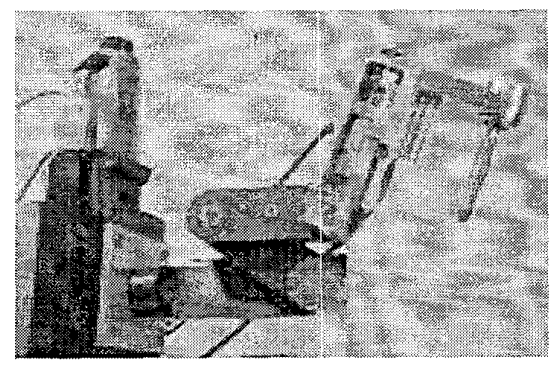

Figure 1: The Steady Hand robot

damped tremor free microsurgery level motions. Immediate applications of this precise robot include eye surgery, microvascular surgery and neurosurgery.

In the targeted application of treating occluded veins, the surgeon hand guides the robot to the treatment site. In addition, the vessels can be imaged using endoscopic images to add non-isotropic gains to favor motion along the desired path. We simulate this situation by using simple compliant motions with the new "steady hand" robot. In these experiments the robot traverses simple paths both under user control and autonomously. An endoscope is mounted on the robot to acquire real time images of the work area. The work area is illuminated appropriately to facilitate image acquisition. The paths to be traversed (lines and simple curves) are easily detectable in images acquired using the endoscope. The user "steady hand" guides the robot by looking at the output of the endoscope on a screen. The robot either a) "favors" compliant motion in the desired direction, b) just complies with the userapplied forces ignoring the detected path. Autonomously, the robot follows the path detected using the images.

Simple strategies are used to favor the desired direction of motion, such as anisotropic gains for compliance. The performance of the robot is judged using metrics such as mean, maximum deviation from the path. These experiments provide performance data for simple augmented procedures such as outlined above. 
They are also indicative of benefits and user acceptance of "steady hand" manipulation

\section{$1.1 \quad$ Previous Work}

\section{Task analysis and Cooperative Manipulation}

Analysis of robot systems operating in tandem with humans and extraction of some information from this cooperation is an active topic of research. Control systems have been proposed by Kazerooni et al ([2-4]). Kosuge et al ([5-7];[8]) have looked at cooperative tasks in industrial environments. Several authors have proposed schemes suitable for cooperative tasks including impedance control strategies ([9], [10]), fuzzy and neural networks ( [11]) etc. Generation of task sequences has been found to be an easier problem; especially for assembly environments and a large body of work are available on this ([12],[13],[14-16]). There is existing previous work in methods for determining force/position control parameters from a human worker's operation $([17,18] ;[19,20])$. Some of these methods are specific to tasks and cannot be applied to other tasks.

In surgical robotics also cooperative control has been a current topic of research. Davies ([21]) and Troccaz $([22,23])$ among others have devised systems that incorporate some level of integration of task information for constrained control.

\section{Evaluation of cooperative performance}

Human performance in surgical procedures has also been studied ([24], [25]). Simulators have also been used to compare/assess surgical skill ([26]). Since complex surgical tasks are difficult to quantify automated performance comparisons in the past have used visual inspection by the experimenter (e.g. [24]) or an observer. Time of completion is often used as a major indicator ([27],[28],[29]).

Peg in hole tasks are commonly used to assess positional accuracy (e.g. [30]). Authors have also previously looked at comparative performance issues ([31]).

Most performance studies report the promise of performance improvement by using a robot over even skilled surgeons, at a cost of increased time of performing the task. They also report ease of use, some requirement for training and increased safety in using the robot.

\section{Manipulator Systems}

Manipulators have been applied to automation of assembly tasks for a longer period and consequently the need for development of efficient user interaction also became evident. More recently, sophisticated systems have been developed to assist, augment human actions in more complex environments, especially in medicine. Currently, they are used to reduce humans involved in a task (i.e. to act as tool, camera holders, or perform a similar routine task) more often than to actually use their superior manipulation abilities. This is similar to the industrial environment where also most of the initial automation consisted of teleoperated devices handling simple tasks or reproduced explicitly taught motions repeatedly.

As in the industrial domain, it has been realized that precision, efficiency, consistency of human performance limits the current practice in several fields of medicine. This is very evident in fields that require high dexterity, long durations of surgery, or high precision of motion.

There have been two approaches to adding automation to medicine. The first, more traditional approach has been to use telemanipulators (e.g. [32], [33], [34]). As in their industrial counterparts, these manipulator systems consist of a master and a slave system. The master robot faithfully reproduces the actions of the slave, often scaled to suit the application. Due to the complex nature of manipulation and difficulty in modeling the environment they perform few automated or "intelligent" functions. The advantages of this approach include a better, more comfortable operating field for the surgeon, scaling of sensory information, and motion commands.

Examples of telemanipulator systems for surgery include the JPL Robot Assisted Microsurgery (RAMS) ([32]) master and slave system, a very sophisticated system for microsurgery and the eye surgery system developed by Hunter et al ([33]) which consists of a master-slave robot system with bi-directional force and motion feedback.

The second, steady hand approach is a hands-on approach. The robot is equipped with a force sensor to sense user interaction. It still acts a slave to user motions but since the user is manipulating the robot as he would the tool, there is no scaling of motion. However, the sensory input driving the motion can be scaled appropriately. The advocates of this approach cite the simplicity, closeness to conventional procedures and therefore ease of use, reduced cost (due to a single manipulator), and improved kinesthetic among the advantages. Several such systems have been built as well.

The new "steady hand" robot ([1]; description below) is a sophisticated hand held manipulator system. The systems developed by Davies ([21]) and Troccaz ([22, 23]) also fall in this category. The LARS ([35]) is a 
camera holding robot designed for laparascopy. Although it was envisioned to be operated with a joystick (and so is not truly a hand held manipulator), LARS has a force sensor built into its end effector that allows hand held manipulator functions.

In both approaches the operator has direct control of the manipulator and its motion. The second approach is more appealing because of its advantages, and also since an experimental platform for this is currently available. This approach will be used for this work.

\section{$2 \quad$ Methods and Materials}

\section{1}

\section{Experimental Setup}

The experimental environment includes the new "steady hand" robot. This is a 7-degree-of-freedom manipulator with $\mathrm{XYZ}$ translation at the base for coarse positioning, two rotational degrees of freedom at the shoulder (the RCM linkage, [36]), and instrument insertion and rotation stages. The instrument stages were not used for this experiment. A force sensor is built in the end-effector. This robot has a remote center of motion and an overall positional accuracy of 10 s of microns.

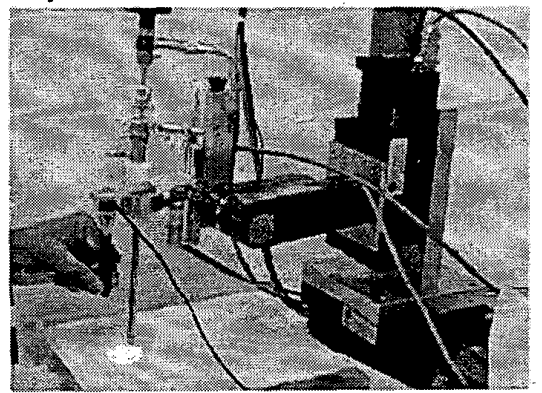

Figure 2: The Experiment Setup

The force sensor is mounted axially with the tool handle that is used for manipulating the tool. The sensor is a small commercially available force sensor (ATI Industrial Automation, NC). The endoscope is mounted on the rotation stage. The force sensor is mounted with its $z$-axis parallel to the instrument insertion stage of the robot.

The robot hardware control runs on a Pentium-II $450 \mathrm{MHz}$ PC with Windows NT operating system. An 8-axis DSP series controller card (PCX/DSP, Motion Engineering Inc, CA) is used to control the robot. The card provides servo control using DSP processor. The PC also houses the ISA force sensor controller. A Matrox Meteor II video capture card is used to digitize the video signal from the endoscope, which is then used for image processing to cornpute the location of the desired feature in the image.

A library of $\mathrm{C}++$ classes has been developed for control purposes. This modular robot control (MRC) library provides Cartesian level control. It includes classes for kinematics, joint level control, command and command table management, sensor and peripheral support, and network support. Some exception and error handling is also built in. An array of sensors including serial and parallel ports, ATI force sensors, joysticks, digital buttons and foot pedals are supported.

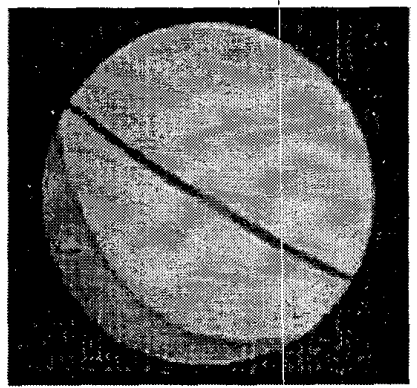

Figure 3: A picture captured from the scope camera, the curve on the left tottom is a part of the endoscope

A simple force proportional velocity controller has been implemented based on the MRC library. User forces at the tool are sensed using the force sensor, biased and resolved the coordinate frame of the robot for force-proportional velocity control. Both user forces and robot velocity are limited for safety. The base joints and the upper joints can be controlled independently by using a foot pedal. Control rates of over $1000 \mathrm{~Hz}$ can be achieved using this controller.

\subsection{Experimental Protocol}

The line and curves drawn on paper are positioned in the working area of the robot and fixed. The robot is then initialized and image processing started. Robot is registered to the camera by detecting a circle on the paper in images taken from several robot positions.
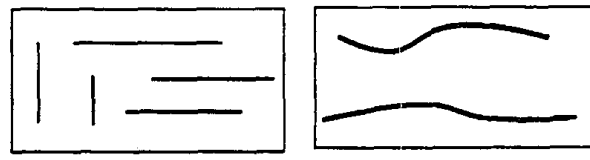

Figure 4: Straight lines (top left)., and simple curves (top right) used in the experiment. 
During path traversal, each acquired image is thresholded, and a search is performed in the center of the image to obtain the gradient and center of the curve in the image. This gradient information is used to compute force gains for the control process in augmented mode. The proportional velocity control described earlier is then used for force proportional velocity control (figure 5).

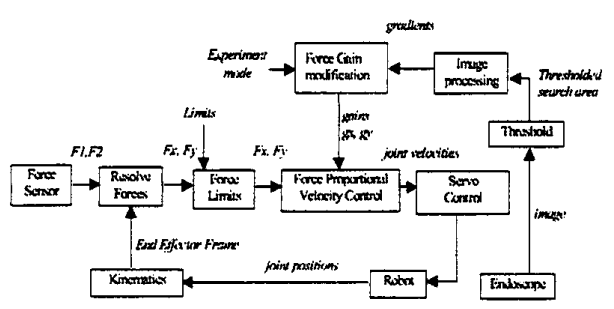

Figure 5: Experimental model

Following three experiments are performed.

a) Autonomous: the robot detects the curve in the images and moves to maintain the curve at the center of the image.

b) Assisted: the robot moves in response to user forces applied at the handle but does not take into account the information obtained from the image processing

c) Augmented: the robot moves in response to user forces but updates the anisotropic gains for control using the information obtained from the endoscope images

For each experiment the trajectory traversed, the location of the curve with respect to the center of image, the time taken, and forces applied are recorded. Two different set of joints, the translation joints in the base, and the rotation joints in the shoulder are used separately for the experiment.

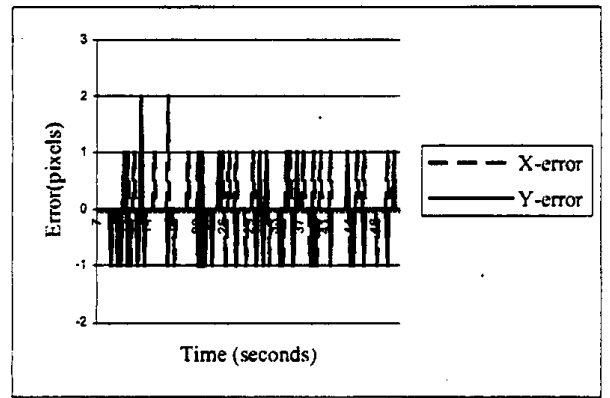

Figure 6: An error profile for automatic tracking for base translation joints $(1$ pixel $=\sim 0.1 \mathrm{~mm}$ ).

\section{Results}

The users are able to trace the straight lines and curves easily in both modes. Augmentation appears to reduce errors. Due to image processing errors, if the gradient detected is not correct, it results in larger errors for the augmented version. Automatic tracking performs superior to other modes in most experiments.

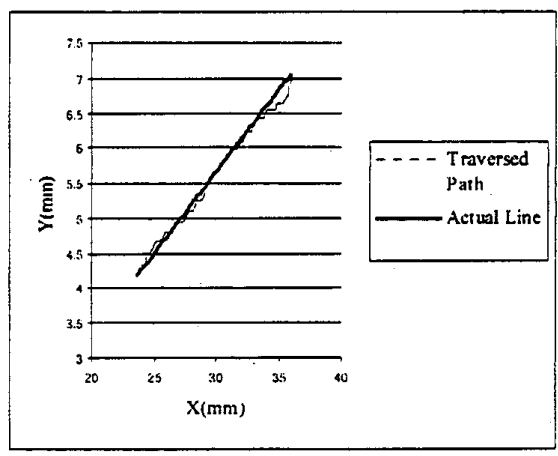

Figure 7: Traced path and actual line for visually guided (augmented) motion for base joints.

Straight lines are the first group of features selected. They simulate vessels to some extent. They are also easy to detect and process in the experimental setup. The robot consistently tracks (e.g. figure 6) to within 1 pixel $(\sim 0.1 \mathrm{~mm})$ average error. Without directional assistance from image processing the assisted experiment errors of up to $1 \mathrm{~mm}$ (figure 8) were commonly observed. The augmented version (figure 7) adds gradient information and reduces the average errors. Detailed results appear in table 1.

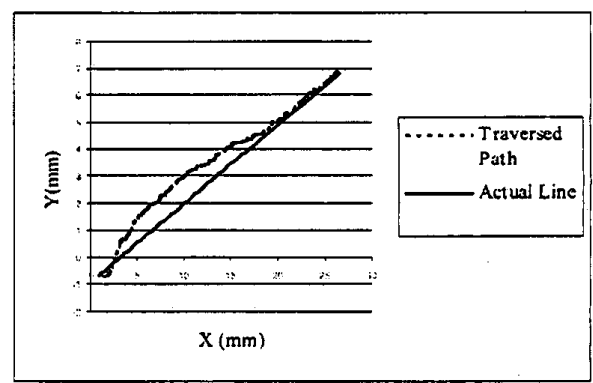

Figure 8: Traced path and actual line for simple compliant motion (assisted series) for base joints.

For simple curves, the gradient information obtained changes often. For automatic tracking, with slow speeds, it is possible to update the gradient information (obtained at about $5 \mathrm{~Hz}$ ) to keep up with the control 
loop (at $1000 \mathrm{~Hz}$ ). The robot autonomously tracks to within an average of $\sim .5 \mathrm{~mm}$ from the center of image. It is more difficult to for users to traverse these curves using simple compliant motion and larger errors, up to $3 \mathrm{~mm}$ are noticed. Using the gradient information in augmented versions reduces the error. Typical Mean and average errors appear in table 2.

The relatively slow rate of image acquisition $(5 \mathrm{~Hz})$, and larger speeds due to user forces might account for the difference between automatic and augmented versions.

\begin{tabular}{|c|c|c|c|c|}
\hline \multirow[t]{2}{*}{ Experiment } & \multicolumn{2}{|c|}{ Translation } & \multicolumn{2}{|c|}{ Rotation } \\
\hline & $\begin{array}{l}\text { Maximum } \\
\text { Error(mm) }\end{array}$ & $\begin{array}{l}\text { Average } \\
\text { EnTor }(m m)\end{array}$ & $\begin{array}{l}\text { Maximum } \\
\text { Error(deg) }\end{array}$ & $\begin{array}{l}\text { Average } \\
\text { Entror(deg) }\end{array}$ \\
\hline Automatic & 0.22 & 0.03 & 0.47 & 0.26 \\
\hline Assisted & 1.6 & 1.1 & 1.04 & 0.61 \\
\hline Augmented & 1.75 & 0.95 & 1.12 & 0.37 \\
\hline
\end{tabular}

Table 1: Typical Mean and Maximum Errors for lines

\section{Conclusions And Scope}

Current experience shows that "steady hand" approach hold promise for improving human performance. With its superior accuracy the robot can help in positioning tasks, very easily. Although image processing in real environments is much harder and information obtained has much more noise. It may still be possible to obtain enough information to assist the user to some extent.

\begin{tabular}{|c|c|c|c|c|}
\hline \multirow{2}{*}{ Experiment } & \multicolumn{2}{|c|}{ Translation } & \multicolumn{2}{|c|}{ Rotation } \\
\hline & $\begin{array}{l}\text { Maximum } \\
\text { Error }(m m)\end{array}$ & $\begin{array}{l}\text { Average } \\
\text { Error }(m \mathrm{~m})\end{array}$ & $\begin{array}{l}\text { Maximum } \\
\text { Error(deg) }\end{array}$ & $\begin{array}{l}\text { Average } \\
\text { ETor(deg) }\end{array}$ \\
\hline Automatic & & 0.34 & 1.54 & 0.54 \\
\hline Assistad & 2.99 & 2.32 & 1.9 & 0.66 \\
\hline Augmented & 7.3 & 0.6 & 1.84 & 0.59 \\
\hline
\end{tabular}

Table 2: Typical Mean and Maximum Errors for curves

Current robotic systems are slower compared to unaided humans (also noticed in [30], [31]) but enhance the performance in these simple tasks. Time is an important consideration in most surgical interventions and robotic systems need further improvement. As also reported by earlier studies ([31]) as well, the augmented "hand held" approach is easy to use and little user training is required to use the system.

\section{Acknowledgements}

We gratefully acknowledge the support of the National Science Foundation under grant \#IIS9801684, the Engineering Research Center grant \#EEC9731478. We thank Aaron Barnes for his help during this work. We also thank Dr. Louis Whitcomb for his helpful suggestions and Andy Bzostek for his valuable help in debugging.

\section{References}

1. Taylor, R.H., et al. A Steady-Hand Robotic System for Microsurgical Augmentation. in MICCAI99. 1999. Cambridge, UK: Springer Verlag.

2. Kazerooni, H. Human/robot interaction via the transfer of power and information signals ..- part $i$ : Dynamics and control analysis. in Proceedings of IEEE International Conference on Robotics and Automation. 1989.

3. Kazerooni, H. Human/robot interaction via the transfer of power and information signals --- part ii: Dynamics and control analysis. in Proceedings of IEEE International Conference on Robotics and Automation. 1989.

4. Kazerooni, H. and G. Jenhwa, Human extenders. Transaction of the ASME: Journal of Dynamic Systems, Measurement and Control, 1993. 115(2B): p. 218-90, June.

5. Kosuge, K., H. Yoshida, and T. Fukuda. Dynamic Control for Robot-Human Collaboration. in IEEE International Workshop on Robot Human Cooperation. 1993.

6. Kosuge, K., Y. Fujisava and T. Fukuda. Control of Robot Directly Maneuvered by Operator. in IEEE International Conference on Robots and Systems. 1993.

7. Kosuge, K., Y. Fujisawa, and T. Fukuda. Control of man-machine system for dexterous manipulation. in Proceedings of the SPIE The Intemational Society for Optical Engineering.

8. Kosuge, K. and N. Kazamura. Control of a robot handling an object in cooperation with a human. in IEEE international workshop on Robot human Communication. 1997.

9. Hogan, N., Impedance control: An approach to manipulation. ASME Journal of Dynamic Systems Measurement and Control, 1985. 107: p. 1--7.

10. Ikeura, R., H. Monden, and H. Inooka. Cooperative Motion Control of a Robot and a Human. in IEEE International Workshop on Robot and Human Coomunication. 1994.

11. Noritsugu, T. and H. Inoue. Master Assisted Cooperative Control of Human and Robot. in IEEE International Workshop on Robot and Human Communication. 1996.

12. Taylor, R.H., The Synthesis of Manipulator Control Programs from Task Level Specifications, . 1976, Stanford University.

13. Prenting, T.O. and R.M. Battaglin, The Precedence Diagram: A tool for analysis in 
assembly line balancing. Journal of Industrial Engineering, 1964. XV(4): p. 208-213.

14. Park, J.H., M.J. Chung, and K.Y. Lim, Acquisition of assmebly constraints without redundancies. IEEE Internation Conference on Robotics and Automation, 1993: p. 69-74.

15. Huang, Y.F. and C.S.G. Lee. An automatic assembly planning system. in IEEE Intl. Conf. on Robotics and Automation. 1990.

16. Baldwin, D.F. and e. al, An Integrated Computer Aid for Generating and Evaluating Assembly sequences for mechanical products. IEEE transactions on robotics and automation, 1991. 7(1): p. 78-94.

17. Asada, $\mathrm{H}$. and $\mathrm{H}$. Izumi, Teaching and Program Generation for the Hybrid Position/Force Control via the Measurement of Human Manipulation Tasks. J. of the Robotics Society of Japan, 1987. 5(6): p. 452459.

18. Asada, H. and B.-H. Yang, Skill Acquisition from Human Experts through Pattern processing of teaching data. Jornal of Robotics Society of Japan, 1990. 8(1): p. 1724.

19. Takahashi, T., H. ogata, and S.-Y. Muto, $A$ method for analyzing human assembly operations for use in automatically generating robot commands. IEEE international conference on robotics and automation, 1993: p. $695-700$.

20. Serge, A.M. and G. DeJong. ExplanationBased Manipulator Learning: Acquisition of Planning Ability Through Observation. in IEEE International Conference on Robotics and Automation. 1985.

21. Davies, B.L., et al., Active Compliance in robotic surgery - the use of force control as a dynamic constraint. Proc Instn Mech Engrs, 1997. 211(H): p. 285-292.

22. Troccaz, J., M. Peshkin, and B.L. Davies. The use of localizers, robots, and synergistic devices in CAS. in Proc. First Joint Conference of CVRMed and MRCAS. 1997. Grenoble, France: Springer.

23. Lavallee, S., et al., Image-Guided Operating Robot: A Clinical Application in Stereotactic Neurosurgery, in Computer-Integrated Surgery, R.H. Taylor, et al., Editors. 1996, MIT Press: Cambridge, Mass. p. 343-352.

24. Salcudean, S.E., S. Ku, and G. Bell Performance measurement in scaled teleoperation for microsurgery. in First joint conference computer vision, virtual realtiy and robotics in medicine and medical robotics and computer-assisted surgery. 1997. Grenoble, France: Springer.
25. Sheridan, T.B. Human factors in Tele inspection and Tele-surgery:Cooperative manipulation under Asynchronous Video and Control Feedback. in MICCAI'98. 1998. Cambridge, MA, USA: Springer-Verlag.

26. OToole, R. and e. al. Assessing Skill and Learning in Surgeons and Medical Students Using a Force Feedback Surgical Simulator. in MICCAI'98. 1998. Cambridge, MA, USA: Springer-Verlag.

27. Starkes, J.L., I. Payk, and N.L. Hodges, Developing a standardized test for the assessment of suturing skill in novice microsurgeons. Microsurgery, 1998. 18: p. 19-22.

28. Tendick, F., S. Bhoyrul, and L. Way, Comparison of Laparoscopic Imaging Systems and Condi-tions Using a Knot Tying Task. Journal of Image Guided Surgery, 1996. 2(1).

29. Kavoussi, L.R., et al. Comparison of robotic versus human laparoscopic camera control. in MRCAS'95. 1995. Baltimore, USA,

30. Das, H., et al., Evaluation of a Telerobotic System to Assist Surgeons in Microsurgery: Computer Aided Surgery, 1999. 4(15): p. 1525.

31. Kumar, R., et al., Robot-assisted microneurosurgical procedures, comparative dexterity experiments, in Society for Minimally Invasive Therapy 9 th Annual Meeting. 1997: Tokyo, Jaban.

32. Schenker, P.S., H.O. Das, and R. Timothy. Development of a new high-dexterity manipulator for robot-assisted microsurgery. in Proceedings of SPIE - The International Society for Optical Engineering: Telemanipulator and Telepresence Technologies. 1995. Boston, MA.

33. Hunter, I.W., Jones L.A., Sagar M.A. Mallinson G.D., Huner P.J., A Teleoperated Microsurgical Robot and Assisted Virtual Environment for Eye Surgery. Presence, 1993. 2(4): p. 265-280.

34. Jensen, P.S., et al., Toward robot assisted vascular microsurgery in the retina. Graefes Arch Clin Exp Ophthalmol, 1997. 235(11): p. 696-701.

35. Taylor, R.H., et al., A Telerobotic Assistant for Laparoscopic Surgery, in IEEE EMBS Magazine Special Issue on Robotics in Surgery. 1995. p. 279-291.

36. Stoianovici, D., et al. An efficient needle injection technique and radiological guidance method for percutaneous procedures. in Proceedings of the First Joint Conference: CVRMed II \& MRCAS III. 1997. 